\title{
Monte Carlo simulations of thermal fluctuations in moderately damped Josephson junctions: Multiple escape and retrapping, switching- and return-current distributions, and hysteresis
}

\author{
J. C. Fenton* and P. A. Warburton \\ London Centre for Nanotechnology, 17-19 Gordon Street, London WC1H OAH, United Kingdom \\ and Department of Electronic and Electrical Engineering, UCL, Torrington Place, London WC1E 7JE, United Kingdom
}

(Received 2 May 2008; revised manuscript received 8 July 2008; published 27 August 2008)

\begin{abstract}
A crossover at a temperature $T^{*}$ in the temperature dependence of the width $\sigma$ of the distribution of switching currents of moderately damped Josephson junctions has been reported in a number of recent papers, with positive $d \sigma / d T$ and $I V$ characteristics associated with underdamped behavior for lower temperatures $T$ $<T^{*}$ and negative $d \sigma / d T$ and $I V$ characteristics resembling overdamped behavior for higher temperatures $T$ $>T^{*}$. We have investigated in detail the behavior of Josephson junctions around the temperature $T^{*}$ by using Monte Carlo simulations including retrapping from the running state into the supercurrent state as given by the model of Ben-Jacob et al. [Phys. Rev. A 26, 2805 (1982)]. We develop discussion of the important role of multiple escape and retrapping events in the moderate-damping regime, in particular considering the behavior in the region close to $T^{*}$. We show that the behavior is more fully understood by considering two crossover temperatures and that the shape of the distribution and $\sigma(T)$ around $T^{*}$, as well as at lower $T<T^{*}$, are largely determined by the shape of the conventional thermally activated switching distribution. We show that the characteristic temperatures $T^{*}$ are not unique for a particular Josephson junction but have some dependence on the ramp rate of the applied bias current. We also consider hysteresis in moderately damped Josephson junctions and discuss the less commonly measured distribution of return currents for a decreasing current ramp. We find that some hysteresis should be expected to persist above $T^{*}$. We highlight the importance, even well below $T^{*}$, of accounting properly for thermal fluctuations when determining the damping parameter $Q$.
\end{abstract}

DOI: 10.1103/PhysRevB.78.054526

PACS number(s): 74.50. $+\mathrm{r}, 74.40 .+\mathrm{k}$

\section{INTRODUCTION}

The Josephson junction system has been extensively studied both theoretically and experimentally. Theoretically, it has been considered a model system for studying escape from a metastable potential well. Experimentally, Josephson junctions have found numerous applications and are presently being used in several quantum-bit implementations. In such experiments, an understanding of the influence of thermal fluctuations is crucial in developing applications. Josephson junctions can be characterized by a damping parameter $Q$. The majority of the large body of previous work in the literature have concentrated on junctions in either the underdamped $(Q \gg 1)$ or the overdamped $(Q \sim 1)$ limit. In this paper, we focus on the intermediate "moderately damped" $(Q \approx 5)$ limit, where thermal fluctuations lead to interesting physical effects.

For strongly underdamped Josephson junctions under the influence of thermal fluctuations, the $I V$ characteristics are hysteretic and the dynamics of switching from the zerovoltage supercurrent state to the finite-voltage resistive phase-slip state are well described by the analysis of Fulton and Dunkleberger, ${ }^{1}$ with a distribution in switching currents as a result of thermal fluctuations. In contrast, overdamped junctions show nonhysteretic behavior, with a finite voltage on the supercurrent branch of the $I V$ characteristic, associated with thermally activated phase diffusion, and thermal fluctuations leading to very much smaller variations in the switching behavior. Phase diffusion in junctions with hysteretic $I V$ characteristics has been discussed by Kautz and Martinis $^{2}$ and is associated with frequency-dependent damping, such that junctions are underdamped at low frequencies but are in the overdamped limit at high frequency.

The temperature dependence of the switching current and the width of its distribution are experimental parameters of much recent interest. Experimental evidence of a crossover in the temperature dependence of the switching current was reported first by Franz et $a l^{3}$ in experiments on small "intrinsic" Josephson junctions (IJJs). They obtained IV curves characteristic of underdamped junctions below a crossover temperature and $I V$ curves characteristic of overdamping above that temperature. More recent experimental papers have reported a crossover in the temperature dependence of the width $\sigma$ of the switching current ${ }^{4-6}$ at a temperature $T^{*}$, with positive $d \sigma / d T$ below $T^{*}$ and negative $d \sigma / d T$ above $T^{*}$. This was associated with a regime of moderate damping. The negative $d \sigma / d T$ region was associated with retrapping of the phase following escape. The low-temperature behavior fits the expectations for underdamped junctions, and the hightemperature behavior resembles previous observations for overdamped junctions with phase diffusion. One might simply explain the crossover from underdamped to overdamped behavior by a temperature-dependent damping $Q$ and this was indeed the suggestion of Franz et al. However, it was demonstrated by Krasnov et al. ${ }^{5}$ that such a crossover should also be expected even for temperature-independent $Q$ if the junctions are in the moderately damped regime $(Q \sim 5)$. Krasnov et al. derived an approximate quantitative formula with $T^{*}=T^{*}(Q)$, implying that $T^{*}$ is a measure of the damping.

Several theoretical treatments of the retrapping process have been presented ${ }^{7-9}$ and the analysis of retrapping was conducted in various ways in the experimental reports of a crossover in $\sigma(T)$. In the analysis in Ref. 4, retrapping was 
assumed to be determined purely by energetic considerations: retrapping is certain to occur where it is energetically expected, below a current $I_{m} \approx 4 I_{c} / \pi Q$, where $I_{c}$ is the (fluctuation-free) critical current of the Josephson junction. For $I>I_{m}$ there is an energy cost $\Delta U_{R}$ to retrapping-in Ref. 4 , retrapping was neglected for $I>I_{m}$. Krasnov et al. ${ }^{5}$ treated retrapping above $I_{m}$ as a thermally activated process, with an energy barrier $\Delta U_{R}$, using the model of Ben-Jacob et al. ${ }^{7}$ Männik et $a l .{ }^{6}$ used Monte Carlo simulations with a resistively and capacitively shunted Josephson junction (RCSJ) model including frequency-dependent damping to determine the probability of thermally induced retrapping following escape.

In this paper, in order to conduct a semianalytic analysis of the multiple escape and retrapping processes, we have adopted the model of Ben-Jacob et al. ${ }^{7}$ We also include the effects of frequency-dependent damping (see Sec. IV A). We develop discussion of the important role of multiple escape and retrapping events in the moderate-damping regime and present results of Monte Carlo simulations showing the variation with experimental parameters of the mean and width of the switching-current distribution. ${ }^{10} \mathrm{We}$ consider the crossover between the lower-temperature conventional underdamped regime and the higher-temperature overdamped regime. Although previous studies have considered a single crossover temperature, we show that, in detail, the change occurs in two stages, with a lower-temperature transition from underdamped behavior to behavior in the crossover regime and a higher-temperature transition from the crossover regime to the higher-temperature overdamped regime. We demonstrate a significant change in the shape of the switching-current distribution around the crossover and study this quantitatively through the skewness parameter.

The process of return from the resistive state in a hysteretic junction is a much less well-studied phenomenon than that of escape. Here we also consider the process of return from the resistive state to the supercurrent state as the current is ramped down and the resulting variation in hysteresis around $T^{*}$. We compare our findings with previous reports in the literature.

In our Monte Carlo simulations, for a current $I$, the probability in a short time interval $\delta t$ of a transition between the metastable and running states is given by $\Gamma_{E}(I) \delta t$ for escape from the metastable state [with $\Gamma_{E}$ given by Eq. (2)] or $\Gamma_{R}(I) \delta t$ for retrapping from the running state [with $\Gamma_{R}$ given below by Eq. (3)]. A bias current is ramped up (or down) at a constant rate in order to generate distributions of switching (or return) currents for junctions with a number of different parameters. We neglect the temperature dependence of the critical current $I_{c}$ and $Q$ in order to emphasize effects due to thermal fluctuations in the junctions. As a bias current is ramped up, a switch is counted when the junction spends more than half the time in the running state over some time period $\tau .{ }^{11}$ Throughout this paper, we use the term "escape" to describe any (possibly short-lived) escape from the instantaneous zero-voltage state and reserve the term "switch" to describe an experimentally measured switch to the running state. Similarly, when describing the behavior as an applied current is ramped down from the critical current, we reserve the term "retrapping" to describe a (possibly short-lived) change from the voltage state to the zero-voltage state and use the term "return" to describe an experimentally measured change from the voltage state to the zero-voltage state.

\section{A. RCSJ model: The underdamped regime}

For a resistively shunted Josephson junction in the absence of fluctuations, escape from the supercurrent state to a state of finite voltage characterized by the junction resistance occurs when the current bias applied to the junction reaches the junction critical current $I_{c}$. At finite temperatures, thermal fluctuations lead to switching at currents below $I_{c}$, and there arises experimentally a distribution in possible values of the switching current. A common experimental configuration is to ramp the current up from zero at a constant rate $d I / d t$. In that case, the probability of a switch in the current range $I$ to $I+d I$ is $p(I) d I$, with ${ }^{1}$

$$
p(I)=\frac{\Gamma_{E}}{d I / d t}\left[1-\int_{0}^{I} p\left(I^{\prime}\right) d I^{\prime}\right],
$$

where $\Gamma_{E}$ is the rate at current $I$ of escape from the supercurrent state.

A mechanical analog for the RCSJ is that of a particle in a washboard potential; it is often used in discussing the dynamics of such junctions. ${ }^{12}$ The height of the corrugations in the untilted washboard is set by the Josephson energy. The current bias corresponds to a tilt of the washboard. The position of the particle along the washboard corresponds to the phase difference across the junction, so that the speed of the particle as it moves in the washboard potential corresponds to the voltage across the junction. As it moves along the washboard, the particle is subject to a viscous damping force which is inversely proportional to the resistance shunting the junction. The strength of the damping can be characterized by a quality factor parameter ${ }^{13} Q=\omega_{P} R C$, where $R$ and $C$ are the resistance and capacitance shunting the junction and $\omega_{P}$ $=\sqrt{2 e I_{c} / \hbar C}$ is the angular frequency of small oscillations at the bottom of the potential well at zero bias. Hysteretic $I V$ characteristics are obtained for $Q \gg 1$ and phase diffusion is obtained for $Q \sim 1$.

\section{B. Characteristic rates}

The rate of thermally activated escape from a minimum in the washboard potential is given by ${ }^{14}$

$$
\Gamma_{E}=a_{t} \frac{\omega_{a}}{2 \pi} \exp \left(-\frac{\Delta U_{E}}{k T}\right),
$$

where $\Delta U_{E}$ is the height of the energy barrier from a washboard potential minimum to the adjacent maximum, $a_{t}$ is a damping-dependent prefactor, and the quantities $a_{t}, \omega_{a}$, and $\Delta U_{E}$ are all current dependent, with $\omega_{a}=\omega_{P}\left[1-\left(I / I_{c}\right)^{2}\right]^{1 / 4}$ and, close to $I_{c}, \Delta U_{E} \approx \frac{4 \sqrt{2}}{3} E_{J}\left(1-I / I_{c}\right)^{3 / 2}$, where the Josephson energy $E_{J}=\hbar I_{c} / 2 e$. Combining Eqs. (1) and (2) gives a characteristic asymmetric distribution of switching currents for such junctions, as shown in Fig. 1(a).

In the underdamped regime, the mean switching current decreases as the temperature increases [Fig. 1(b)] because 

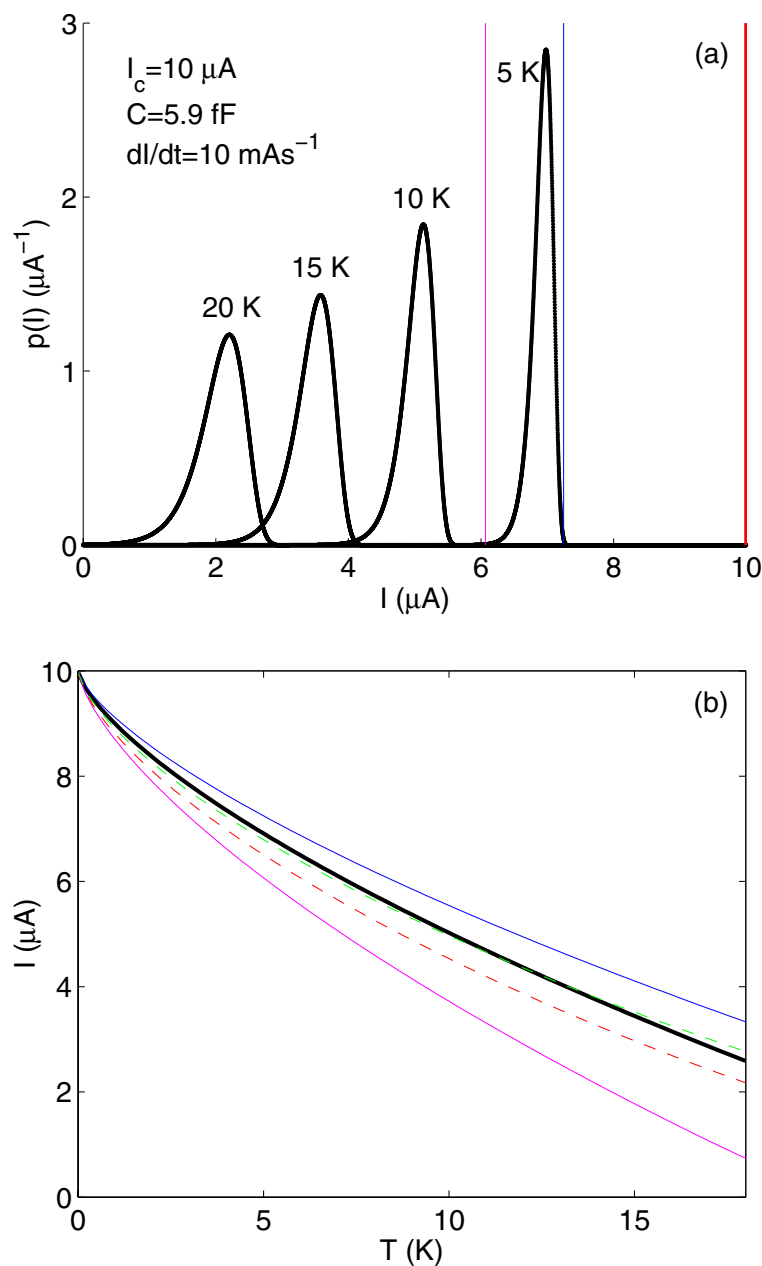

FIG. 1. (Color online) (a) Calculated underdamped thermally activated $p(I)$ distributions at a number of temperatures. The parameters indicated are used for subsequent figures unless otherwise stated; these parameters might be typical for an IJJ (Ref. 15). The vertical lines (pink and blue online) show the boundaries of the distribution at $5 \mathrm{~K}$, within which $99.99 \%$ of switching events occur. The thick vertical line (red online) shows the critical current. (b) Variation with temperature of the mean switching current (thick black line) and the top (blue online) and bottom (pink online) of the switching-current distribution. The lower and upper broken lines (red and green online) show respectively $I\left(\Gamma_{E}=\Gamma_{I}\right)$ and $I\left(\Gamma_{E}\right.$ $\left.=10 \Gamma_{I}\right)$.

larger thermal fluctuations enable escape from the washboard minimum at a lower current. The width of the switchingcurrent distribution may be shown to depend on temperature as $\sigma \sim T^{2 / 3}$.

Thermal fluctuations can also cause retrapping of a particle which has escaped from a potential well. Ben-Jacob et $a l{ }^{7}$ obtained an analytic formula for the rate $\Gamma_{R}$ of this retrapping in the limit $Q \gg 1$. The retrapping rate is strongly dependent on the damping through $Q$ and is given by

$$
\Gamma_{R}=\frac{I-I_{\mathrm{r}}}{I_{c}} \omega_{P} \sqrt{\frac{E_{J}}{2 \pi k T}} \exp \left[-\frac{E_{J} Q^{2}\left(I-I_{\mathrm{r}}\right)^{2}}{2 k T I_{c}^{2}}\right],
$$

where $I_{\mathrm{r}}=I_{\mathrm{r}}(Q) \approx 4 I_{c} / \pi Q$. Rewriting this in the form $\Gamma_{R}$ $\sim \exp \left(-\Delta U_{R} / k T\right)$ defines an energy barrier $\Delta U_{R}$ for retrapping. ${ }^{5}$ Equation (3) has been applied in the literature ${ }^{5}$ in the regime of moderate damping $Q \gtrsim 5$, and we consider here in further detail the application of the model in that regime.

It is instructive to define a normalized current-ramp rate $\Gamma_{I} \equiv \frac{1}{I} d I / d t$. Equation (1) can then be rewritten as

$$
p(I)=\frac{\Gamma_{E}}{\Gamma_{I}} \frac{1-\int_{0}^{I} p\left(I^{\prime}\right) d I^{\prime}}{I} .
$$

For small currents, $\Gamma_{E} \ll \Gamma_{I}$, so $p(I)$ is small. As the current is increased toward the current $I_{E I}$, at which $\Gamma_{E}=\Gamma_{I}$, the first quotient in Eq. (4) increases. Therefore, as the current increases further, the numerator of the second quotient ${ }^{16}$ begins to reduce from 1 to zero. The maximum in $p(I)$ therefore occurs for $\Gamma_{E} \gtrsim \Gamma_{I}$. The dashed lines in Fig. 1(b) show the currents at which $\Gamma_{E}=\Gamma_{I}$ and $\Gamma_{E}=10 \Gamma_{I}$. The exact ratio $\Gamma_{E} / \Gamma_{I}$ at the maximum in $p(I)$ is temperature dependent: at 5 $\mathrm{K}$, the peak in the switching current lies at a higher current than the current at which $\Gamma_{E}=10 \Gamma_{I}$, whereas at $15 \mathrm{~K}$, the peak in the switching current lies at a lower current than the current at which $\Gamma_{E}=10 \Gamma_{I}$.

\section{MULTIPLE ESCAPE-RETRAPPING REGIME}

As the current is increased from zero, the three characteristic rates $\Gamma_{E}, \Gamma_{R}$, and $\Gamma_{I}$ vary. Figure 2(a) shows the variation in these three rates when $Q=200$, i.e., for an underdamped junction. At very low currents, the retrapping rate is much larger than $\Gamma_{E}$ and $\Gamma_{I}$. Also, since $\Gamma_{E} \ll \Gamma_{I}$, no escape events occur. When the current is increased to around $I_{E I}$, an escape event becomes likely, but for $I \gtrsim I_{E I}$ the retrapping rate is very much smaller than the escape rate. Therefore retrapping is negligible in the case illustrated in Fig. 2(a). As we will see, an important current is the current $I_{E R}$, at which $\Gamma_{E}=\Gamma_{R}$. In Fig. 2(a), $I_{E R}=0.31 \mu \mathrm{A}$ and $I_{E R}<I_{E I}$.

Figure 2(b) shows the variation in the three characteristic rates for a more heavily damped junction. The escape rate is only weakly dependent on $Q$ through the prefactor $a_{t}$ [Eq. (2)]. The retrapping rate is exponentially dependent on $Q$ [Eq. (3)]; it is much larger in Fig. 2(b) than in Fig. 2(a) and $I_{E R}>I_{E I}$. For currents $I \sim I_{E I}$, the retrapping rate $\Gamma_{R}$ is now much larger than $\Gamma_{E}$. Escape events occur for $I \gtrsim I_{E I}$, but retrapping occurs shortly afterwards. The particle moves down the washboard in fits and starts and the time-averaged voltage across the junction is nonzero-this state can be called a region of phase diffusion. ${ }^{17}$ As the current increases, the escape and retrapping rates become more and more similar, so there is a gradual increase in the time-averaged voltage. Figure 3 shows for the same values of $Q$ and $T$ as in Fig. 2(b), a simulation of jumps between the supercurrent (zero voltage) and running (resistive) states at three currents close to $I_{E R}$. In Fig. 3(a), $I<I_{E R}$ and the junction spends most of the time in the zero-voltage state. At $I \sim I_{E R}$ [Fig. 3(b)], escape events and retrapping events are expected in similar proportion and the junction spends a similar amount of time in the zero-voltage and escaped states. The time-averaged voltage across the junction becomes a significant fraction of 

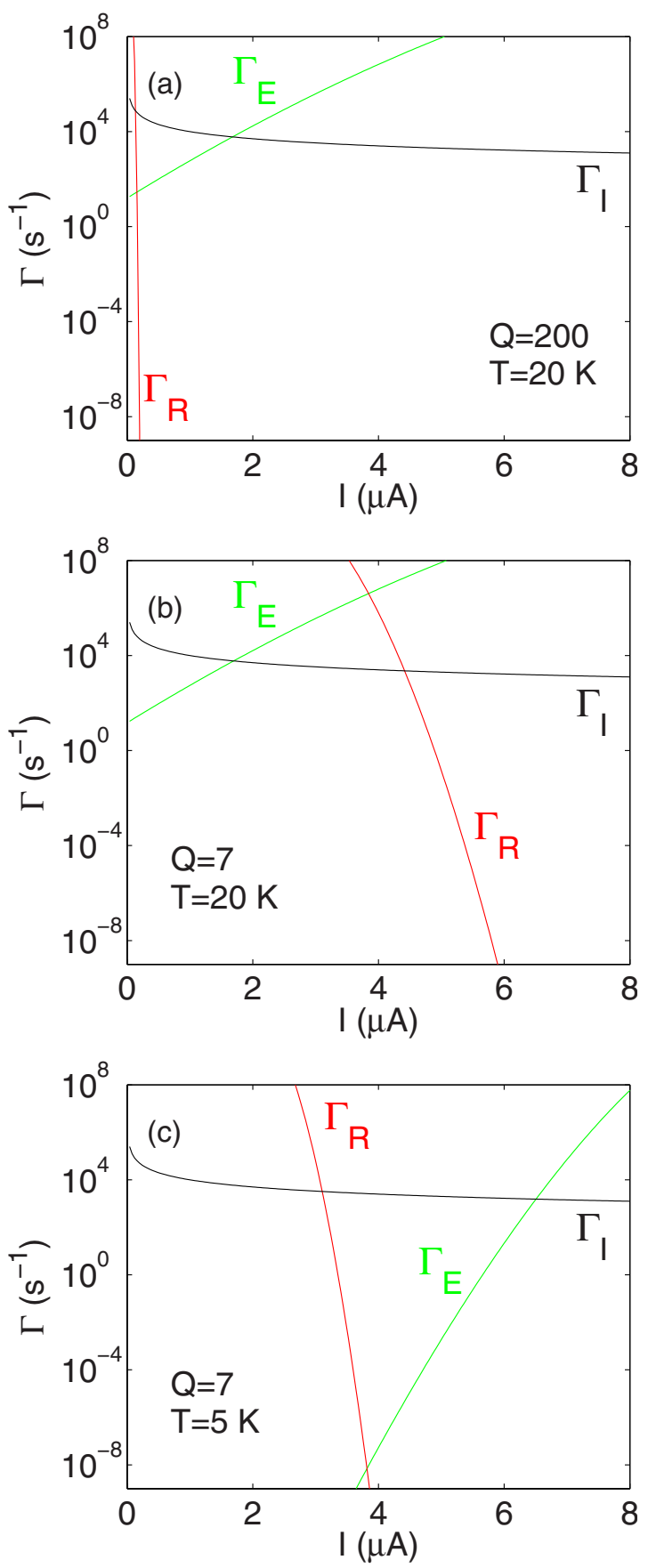

FIG. 2. (Color online) Variation in characteristic rates with current. The different panels show the effect of variations in temperature and $Q$.

the fully switched voltage, so an experiment is likely to measure a switch event. As the current is increased further above $I_{E R}$, any retrapping event will be followed quickly by an escape event, so the junction spends almost all of its time in the running state, as Fig. 3(c) shows.

For the junction parameters corresponding to Fig. 2(b), the junction switches around $I_{E R}>I_{E I}$, so the switching current is greater than the switching current in the underdamped case. In other words, counterintuitively, thermal fluctuations suppress the switching current less in the multiple escape-
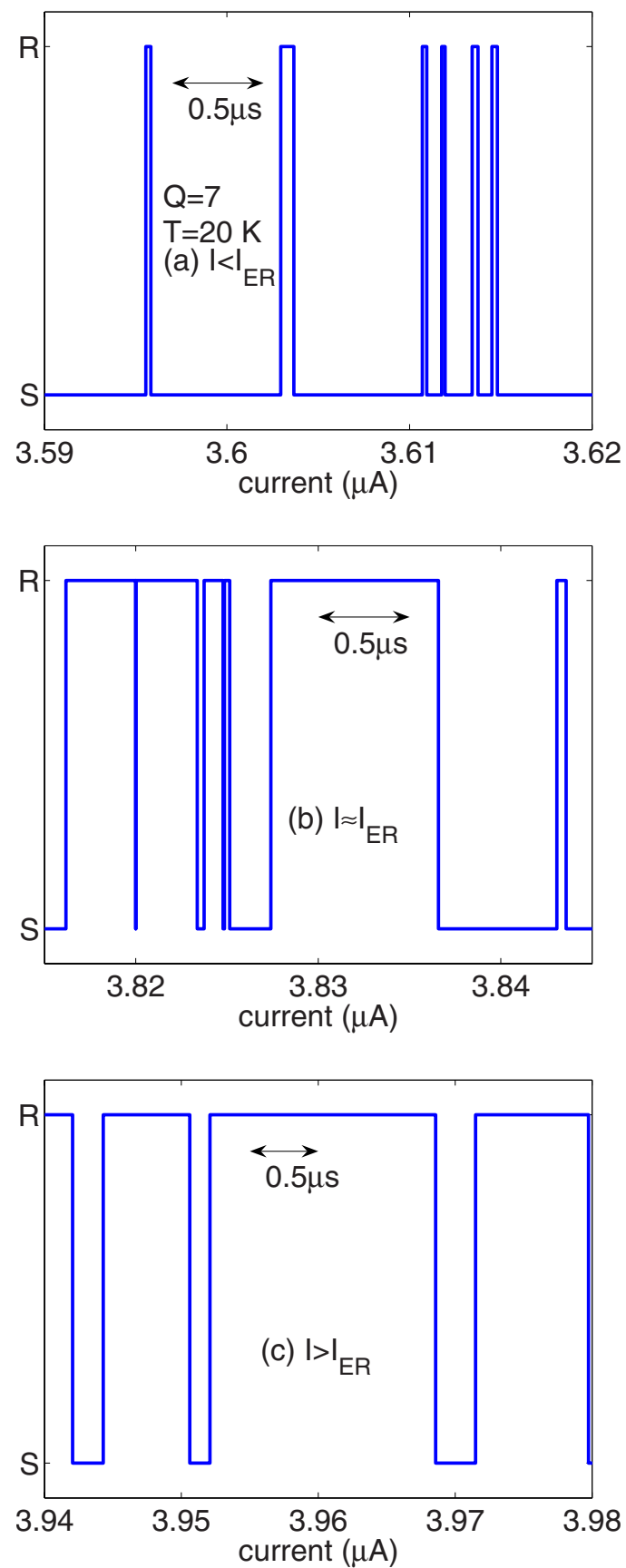

FIG. 3. (Color online) Switching between zero-voltage supercurrent state $(\mathrm{S})$ and the resistive running state $(\mathrm{R})$ at three currents close to $I_{E R}=3.8417 \mu \mathrm{A}$ in a representative simulation for the junction parameters shown in Fig. 2(b). The instantaneous voltage in the running state well above the retrapping current is given by $I R$, where $R$ is the relevant resistance shunting the junction.

retrapping regime than in the conventional underdamped thermally activated switching regime. ${ }^{18}$

\section{TEMPERATURE DEPENDENCE, THE CROSSOVER REGIME, AND $T^{*}$}

Figures 2(b) and 2(c) show the variation in the characteristic rates for two different temperatures with $Q=7$. At the 


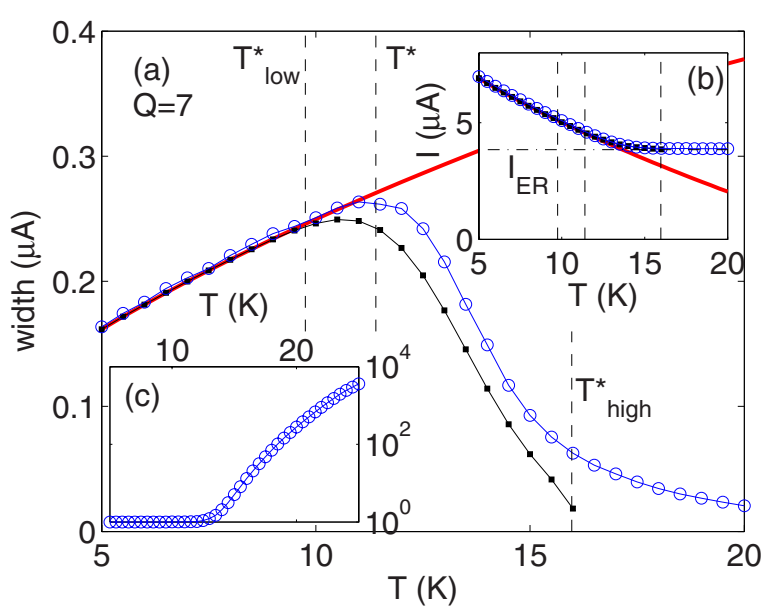

FIG. 4. (Color online) (a) Variation in standard deviation of switching distribution for $Q=7$ with temperature. (b) Variation in mean switching current with temperature for $Q=7$. Shown are simulation results (open circles, with a line to guide the eye; blue online), the full underdamped thermal (thick line, red online), and the underdamped thermal distribution truncated to above $I_{E R}$ (small closed squares, with a black line to guide the eye). The dash-dotted line shows $I_{E R}$ and $T^{*}$ is defined by the maximum in the simulated distribution width. (c) Variation with temperature of the mean number of escape events before a switch is counted in simulations. In the underdamped thermal case, a single escape event would lead to a switch. Above $T_{\text {high }}^{*}$, the distribution in this value is smaller than the symbols. Simulated distributions were based on 25000 switching events.

lower temperature, $5 \mathrm{~K}$ [Fig. 2(c)], $I_{E I}>I_{E R}$, so for $\Gamma_{E} \sim \Gamma_{I}$, the retrapping rate is smaller than the escape rate. Therefore, retrapping after escape does not occur, and the conventional underdamped thermal activation behavior is obtained. Conversely, at a higher temperature, $20 \mathrm{~K}$, [Fig. 2(b)], $I_{E I}<I_{E R}$, and so there are multiple escape and retrapping events, as described earlier. Note that $I_{E R}$ is approximately unchanged as temperature varies at constant $Q$. This is expected by inspection of Eqs. (2) and (3). Ignoring corrections of logarithmic order, $\Delta U_{E}\left(I_{E R}\right)=\Delta U_{R}\left(I_{E R}\right)$ determines $I_{E R}$, where $\Delta U_{E}$ and $\Delta U_{R}$ are both independent of temperature and hence $I_{E R}$ is independent of temperature too.

The results of simulations of these dynamics over a broader range of temperatures are shown in Fig. 4. In Fig. 4(a), the width of the distribution follows the conventional underdamped thermal behavior $\left(\sigma \sim T^{2 / 3}\right)$ at lower temperatures, passes through a maximum, and then falls at higher temperatures, matching experimental observations. In the previous experimental reports, ${ }^{4-6}$ a characteristic temperature $T^{*}$ was defined as the temperature at which the maximum in $\sigma(T)$ occurs. At low temperatures, a single escape event results in a switch being counted [Fig. 4(c)] and the mean of the distribution follows the conventional underdamped thermal behavior [Fig. 4(b)]. At around $T^{*}$, the mean switching current flattens out and reaches an approximately constant value $I \approx I_{E R}$ well above $T^{*}$. For higher temperatures a significant number $\left(\sim 10^{3}-10^{4}\right.$ above $\left.25 \mathrm{~K}\right)$ of escape events occurs before a switch is counted. The shape of the switching distribution also changes as the temperature is in-

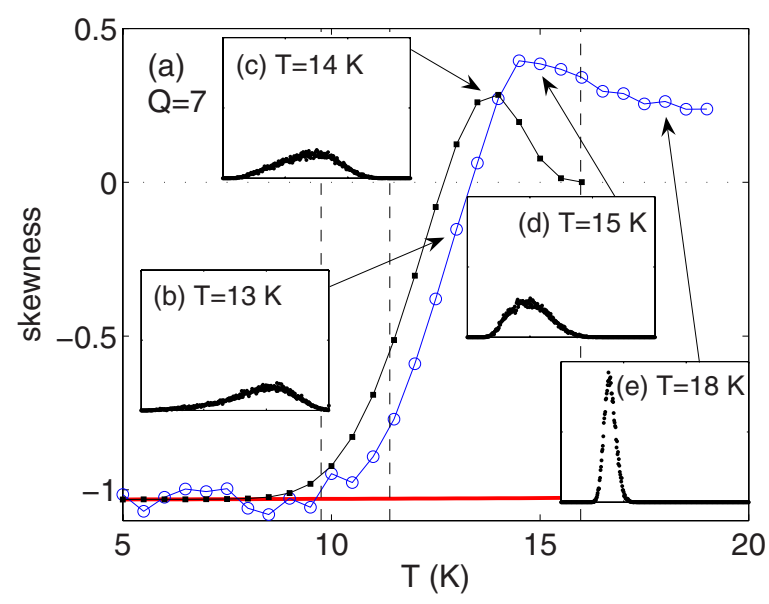

FIG. 5. (Color online) (a) The skewness of the switching distribution, negative for conventional underdamped distributions, becomes positive around $T_{\text {high. }}^{*}$. Points and lines are as described in Fig. 4. (b)-(e) Switching distributions at selected temperatures. Note that the scales on these insets are the same, with the scale on the horizontal current axis running between 3.5 and $5 \mu \mathrm{A}$ and the scale on the vertical $p(I)$ axis running from 0 to $10 \mu \mathrm{A}^{-1}$. Simulated distributions were based on 25000 switching events.

creased. Figures 5(b)-5(e) show that the shape departs from that shown in Fig. 1. The skewness (the ratio of the third moment about the mean to the standard deviation) gives a simple one-parameter description of the shape of the distribution; a symmetrical distribution has zero skewness. The skewness of the underdamped thermal distribution is around -1 over the range of temperature shown in Fig. 4. Figure 5 (a) shows the variation in the skewness of the simulated distribution around $T^{*}$. The skewness of the distribution begins to depart from its thermal value somewhat below $T^{*}$, becoming progressively less negatively skewed and then positively skewed, passing through a maximum and then beginning to level out at around the same temperature as the width begins to level out and as the mean switching current levels off.

From these simulations, we identify three different regimes of behavior. At low temperatures, conventional thermal underdamped behavior is observed. At some higher temperature below $T^{*}$, the skewness of the distribution and in detail also the width and the mean depart from the underdamped thermal values. Above this temperature, the skewness and width vary rapidly. At a higher temperature, there is a crossover to a different regime in which the mean switching current is approximately constant and the skewness and width are slowly decreasing as the temperature is increased. To describe this behavior, we label the two boundaries between these three regimes $T_{\text {low }}^{*}$ and $T_{\text {high }}^{*}$, where $T_{\text {low }}^{*}<T^{*}$ $<T_{\text {high }}^{*}$.

To arrive at a quantitative definition for $T_{\text {low }}^{*}$, we note that retrapping only has a significant effect on the dynamics when there are escapes at currents $I<I_{E R}$. Therefore, for parameters where there are no escapes for $I<I_{E R}$, the switching distribution does not depart from the conventional underdamped thermal distribution. We define $T_{\text {low }}^{*}$ quantitatively as the temperature at which $I_{E R}$ coincides with the bottom of the 
conventional thermally activated underdamped switching distribution [see also the lower line (pink online) in Fig. 1], where we define the bottom $I_{b}$ and top $I_{t}$ of the distribution by $\int_{0}^{I_{b}} p(I) d I=f_{p}$ and $\int_{I_{t}}^{I_{c}} p(I) d I=f_{p}$, where $0<f_{p} \ll 1$, with $f_{p}$ $=0.0005$. Figure 6(a) shows a simulated switching-current distribution at $T_{\text {low }}^{*}$ and a comparison of $\Gamma_{E}, \Gamma_{R}$, and $\Gamma_{I}$ as a function of current.

As the temperature increases, the current $I_{E I}$ decreases. For temperatures $T>T_{\text {low }}^{*}$, escape events occur for $I_{E I} \lesssim I$ $\lesssim I_{E R}$ and are followed by retrapping events; they do not result in the count of a switch. Figure 6(b) shows for a temperature $T>T_{\text {low }}^{*}$ a comparison of the characteristic rates. It also shows that the simulated switching distribution begins to depart from the conventional underdamped thermally activated switching distribution.

For $T>T_{\text {low, }}^{*}$, escape events leading to switching only occur at currents $I \gtrsim I_{E R}$. When a part of the underdamped thermal distribution lies at $I \gtrsim I_{E R}$, one might naively expect that the width, mean, and shape of the switching distribution would be approximately the same as the width, mean, and shape of the part of the underdamped thermal distribution lying at $I>I_{E R}$. The mean, width, and skewness of the part of the underdamped thermal distribution lying above $I_{E R}$ ("the truncated distribution") are shown in Figs. 4 and 5. The mean in the simulations closely matches the mean of the truncated underdamped thermal distribution. The temperature dependence of the width and the skewness in the simulations also follows the respective variations in the width of the truncated distribution up to temperatures approaching the temperature at which $I_{E R}$ coincides with the top of the conventional thermally activated underdamped switching distribution [see also the upper line (blue online) in Fig. 1]. We define this latter temperature as $T_{\text {high }}^{*}$ [see Fig. 6(c)]. The shape of the part of the underdamped thermal distribution with $I>I_{E R}$ largely determines the shape of the switching distribution for $T_{\text {low }}^{*}$ $<T<T_{\text {high }}^{*}$ : the conventional thermal behavior is followed only below $T_{\text {low }}^{*}$, but the variations in the mean and width of the distributions remain analytically determinable for temperatures up to $T_{\text {high }}^{*}$.

The departure of $I_{\mathrm{sw}}$ from the conventional thermal activated behavior above $T_{\text {low }}^{*}$ and the approach to an asymptotic value is in agreement with experimental results reported by Franz et al. ${ }^{3}$ in which a plateau in $I_{\text {sw }}(T)$ was observed above a crossover temperature, followed by a fall at higher temperatures. In one sample, however, they observed an increase in $I_{\mathrm{sw}}$ above the crossover temperature. These observations may be explained by considering temperature variation in $I_{c}$ and $Q$. The Ambegaokar-Baratoff relation ${ }^{19}$ suggests a significant decrease in $I_{c}$ for $T>T_{c} / 2$. A reduction in $I_{c}$ as $T$ increases would lead to a fall in the switching current, whereas a decrease in $Q$ could lead to an increase in the switching current. One might expect a reduction in $Q$ at higher temperatures as quasiparticle conductivity increases. This change will be sample dependent; for a typical IJJ sample, the resistance may fall by a third from low temperature to $T_{c}$. However, if the shunt resistance is dominated by the environmental impedance, $Q$ is expected to be approximately $T$ independent.

Since, as the temperature increases above $T_{\text {low }}^{*}$, the simulated distribution becomes progressively less negatively
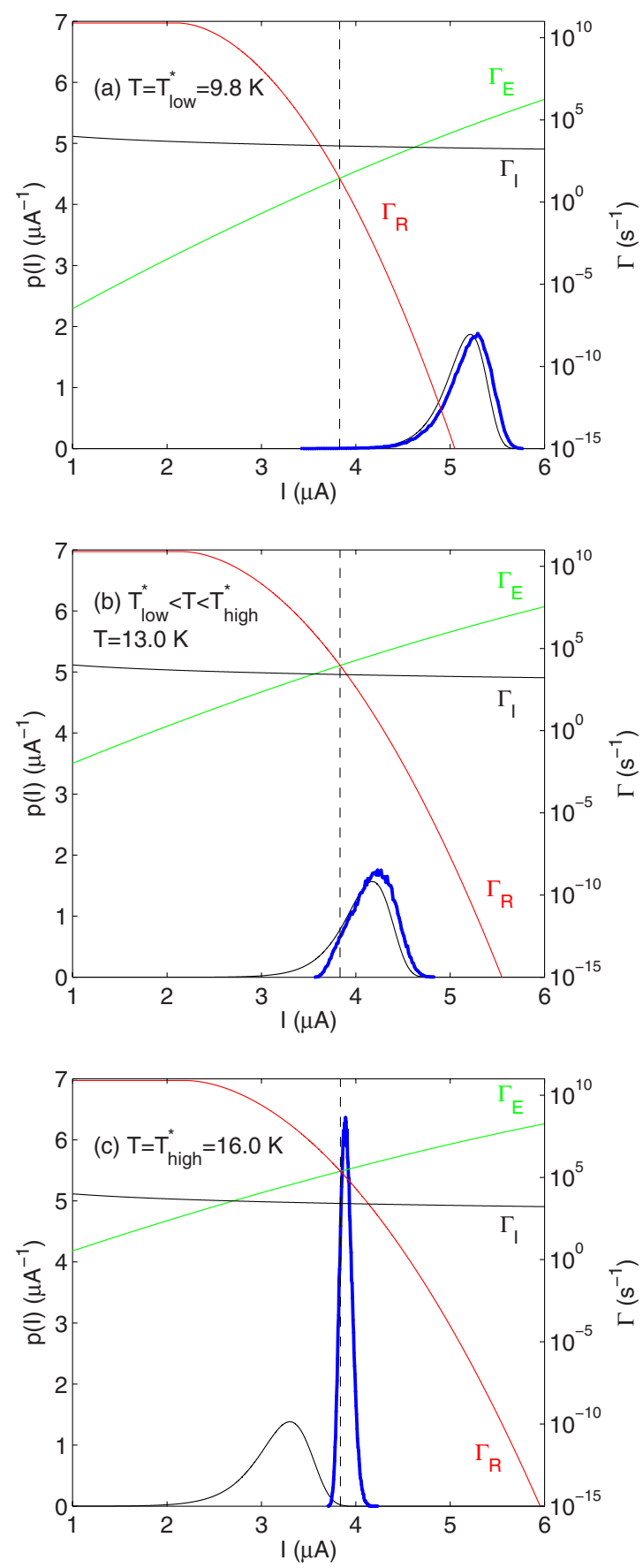

FIG. 6. (Color online) Variation in characteristic rates with current at three temperatures for $Q=7$, along with the switching distribution. The heavy points (blue online) show the simulated switching distribution. The lower black curve shows the underdamped thermally activated switching distribution. The broken black line depicts the current $I_{E R}$. (a) $T=T_{\text {low }}^{*}=9.8 \mathrm{~K} ; I_{E R}$ coincides with the bottom of the thermal distribution. (b) $T=13.0 \mathrm{~K}$. (c) $T=T_{\text {high }}^{*}$ $=16.0 \mathrm{~K} ; I_{E R}$ coincides with the top of the thermal distribution. Simulated distributions were based on 100000 switching events.

skewed and becomes positively skewed around $T_{\text {high }}^{*}$, the temperature variation of the skewness provides a straightforward experimental way to determine $T_{\text {low }}^{*}$ and $T_{\text {high }}^{*}$. As far as we are aware, no systematic experiments investigating varia- 
tions in the shape of the switching distribution around $T^{*}$ have yet been reported.

For $T \gtrsim T_{\text {high }}^{*}$, the behavior is not associated with the underdamped thermal distribution; we will not discuss this behavior in detail. Figure 4(b) shows that for $T>T_{\text {high }}^{*}$, the mean switching current approaches $I_{E R}$. Since $I_{E R}$ is a function of $I_{c}$ and $Q$, the determination of $I_{E R}$ from the switching current at $T \gtrsim T_{\text {high }}^{*}$ provides an additional experimental probe for the determination of $I_{c}$ and $Q$ and a consistency check for the derivation of $Q$ either from $T^{*}$ or from a ratio of the switching and return currents. In addition, the expectation that the mean of the switching distribution at temperatures above $T_{\text {high }}^{*}$ does not vary with temperature for constant $Q$ means that this measurement might be used as an experimental probe for determining whether $Q$ varies with temperature. We return later to discuss how the presence of frequency-dependent damping affects this plateau.

For $T>T_{\text {low }}^{*}$, the behavior shows some similarities to the extensively studied phenomenon of phase diffusion; it also shows some differences. The time-averaged voltage across the junction is finite below the switching current, as expected for conventional phase diffusion. However, for $I<I_{E I}$, the time-averaged voltage across the junction is identically zero with a high probability. ${ }^{20}$ This behavior contrasts with a phase-diffusion regime considered by Ivanchenko and Zil'berman ${ }^{21}$ and others in which a finite phase-diffusion resistance persists even at currents $I \rightarrow 0$. For $I>I_{E I}$, the timeaveraged junction voltage is finite, but this phase-diffusion regime also differs physically from that analyzed by Ivanchenko and Zil'berman. ${ }^{21}$ Ivanchenko and Zil'berman considered that each escape would lead to a phase shift of only $2 \pi$, whereas in our model the phase shifts are of order $\omega_{P} / \Gamma_{R} \gg 1$. Kautz and Martinis ${ }^{2}$ previously showed that the presence of multiple- $2 \pi$ phase shift-escape events has an appreciable effect on the phase-diffusion voltage. This is associated with the time $\sim 1 / \omega_{P}$ needed after the energy barrier is exceeded for the instantaneous voltage to increase from zero to its steady value. In previous experiments on moderately damped junctions, Krasnov et al..$^{5}$ did not observe any phase-diffusion voltage above $T^{*}$ until well above $T^{*}$. This appears to conflict with our understanding from our analysis. However, the explanation might simply be that the phasediffusion voltage was too small to be measurable.

\section{Ramp-rate dependence}

Since the crossover temperatures are dependent on the shape of the thermal distribution and the shape of the thermal distribution is dependent on the current-ramp rate, the crossover temperatures are also dependent on the current-ramp rate. Figure 7 shows the variation with current-ramp rate of calculated values of $T_{\text {low }}^{*}$ and $T_{\text {high }}^{*}$ and the temperature $T^{*}$ at which the maximum in the width occurs. All these values were determined from the truncated thermal distribution. Also shown is $T_{I E R}^{*}$, the temperature at which $I_{E R}=I_{E I}$, which in this analysis is the most natural definition for a single crossover temperature. For a ramp rate $d I / d t=10^{-4} \mathrm{~A} \mathrm{~s}^{-1}$, we find $T_{\text {low }}^{*}=8.2 \mathrm{~K}$ and $T_{\text {high }}^{*}=12.2 \mathrm{~K}$, whereas for a ramp rate $d I / d t=10^{-1} \mathrm{~A} \mathrm{~s}^{-1}$, we find $T_{\text {low }}^{*}=10.8 \mathrm{~K}$ and $T_{\text {high }}^{*}$

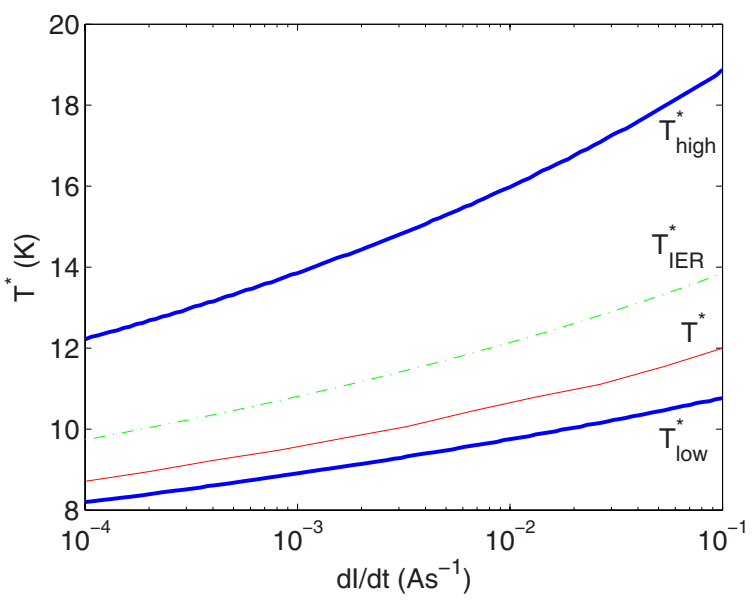

FIG. 7. (Color online) Calculated variation in $T_{\text {low }}^{*}$ and $T_{\text {high }}^{*}$ with ramp rate, with $f_{p}=0.0005$ and $T_{I E R}^{*}$, the temperature at which $\Gamma_{E}, \Gamma_{R}$, and $\Gamma_{I}$ are equal at some value of current. Also shown is the temperature at which the maximum width of the truncated ( $I$ $\left.>I_{E R}\right)$ distribution lies.

$=18.9 \mathrm{~K}$. Thus, as the simulations reported in Fig. 8 show, varying the ramp rate can have a significant effect on $T^{*}$; this dependence was not recognized in previous reports of the crossover. Figure 8(a) shows simulations of the variation in the width of the switching distribution with temperature for a number of current-ramp rates. In the range shown, for $d I / d t=10^{-4} \mathrm{~A} \mathrm{~s}^{-1}$ the temperature is above $T_{\text {low }}^{*}$, whereas for $d I / d t=10^{-1} \mathrm{~A} \mathrm{~s}^{-1}$ the temperature ranges from below $T_{\text {low }}^{*}$ to above $T_{\text {high }}^{*}$. This difference between the two ramp rates leads to a marked difference in the temperature variation of the width.

Experimentally, probably the most straightforward measurement for investigating these effects would be to keep $T$ fixed and vary $d I / d t$. Figure 8 (b) shows a simulation of this procedure-there may be a marked difference in the variation in the width with ramp rate depending on the temperature of the measurement. For $T=9 \mathrm{~K}$, the temperature is at or below $T_{\text {low }}^{*}$ for the whole range of ramp rates, whereas for $T=18 \mathrm{~K}$, the temperature is at or above $T_{\text {high }}^{*}$ for the whole range of ramp rates. For intermediate temperatures, increasing the ramp rate from $10^{-4}$ to $10^{-1} \mathrm{~A} \mathrm{~s}^{-1}$ moves the $T^{*}$ values so that the temperature is close to $T_{\text {high }}^{*}$ at the lowest ramp rate and close to $T_{\text {low }}^{*}$ at the highest ramp rate. This ramp-rate dependence of the width could be used to determine $Q$. As the ramp rate is varied at a constant temperature, $Q$ remains fixed, but $T^{*}$ varies and so the width of the distribution varies, particularly when $T^{*}$ becomes close to the experimental temperature. This allows $Q$ to be determined, at each experimental temperature, by fitting to simulations.

\section{RETURN CURRENT $I_{\mathrm{r}}$, HYSTERESIS, AND FREQUENCY-DEPENDENT DAMPING}

In previous papers ${ }^{3,5}$ it was noted that, although the temperature at which the hysteresis in the $I V$ characteristic disappeared was around $T^{*}$, there was some difference between the two values. Motivated by this discrepancy, we consider 

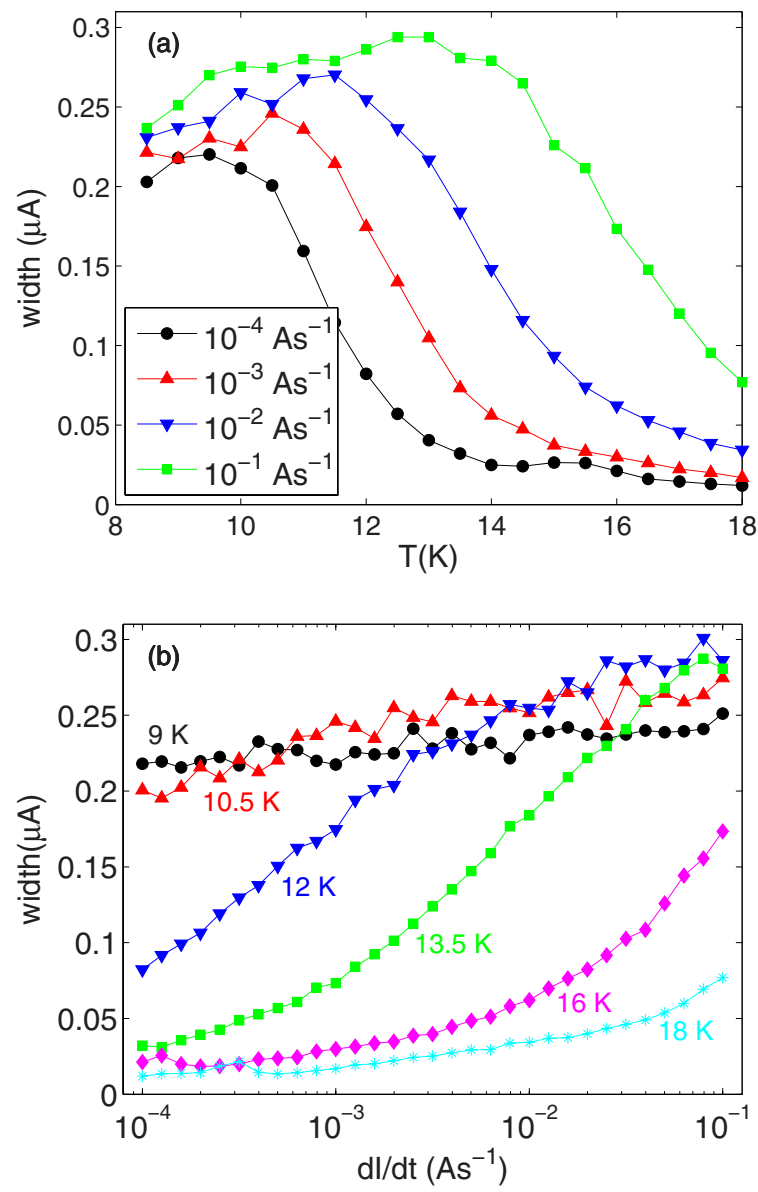

FIG. 8. (Color online) (a) Variation in width with temperature around $T^{*}$ for various ramp rates. (b) Variation in width with ramp rate for various temperatures close to $T^{*}$. Each data point corresponds to a simulated distribution based on 1000 switching events. Departures from a smooth trend are visible as a result of statistical fluctuations. The lines are guides to the eye.

here in detail the variation in hysteresis around $T^{*}$.

In the RCSJ model in the absence of fluctuations, as an applied current is ramped down from $I_{c}$ toward zero, return from the quasiparticle branch occurs at a current $I_{R}$ $\approx 4 I_{c} / \pi Q$ for $Q \gtrsim 3 .{ }^{22}$ Thermal fluctuations lead to retrapping when $\Gamma_{R} \sim \Gamma_{I}$, at currents $I_{\mathrm{r}}>I_{R}$. As Fig. 9 shows, there is some distribution in the value at which return occurs, and the mean and peak of the distribution lie below the current at which $\Gamma_{R}=\Gamma_{I}$. The width of the return distribution may be shown to vary with $T$ as $\sigma \sim T^{1 / 2} \cdot{ }^{23}$ Experimentally, measurements of return distributions are more likely than switchingdistribution measurements to be affected by heating and this complicates analysis of the temperature dependence. To our knowledge, the only report in the literature of an experiment in which the distribution of return currents was measured has been given by Castellano et al. ${ }^{24}$

A measure of the hysteresis of the junction is given by $I_{\mathrm{r}} / I_{\mathrm{sw}}$ and, experimentally, ${ }^{5} I_{\mathrm{r}} / I_{\mathrm{sw}}$ is sometimes used to infer $Q$ through the approximation $I_{\mathrm{r}} / I_{\mathrm{sw}} \approx I_{R} / I_{c}=4 / \pi Q$. However, for moderately damped Josephson junctions, the ratio $E_{J} / k T$ is often sufficiently small that thermal fluctuations cause significant departures of $I_{\mathrm{r}}$ from $I_{R}$ and $I_{\mathrm{sw}}$ from $I_{c}$.
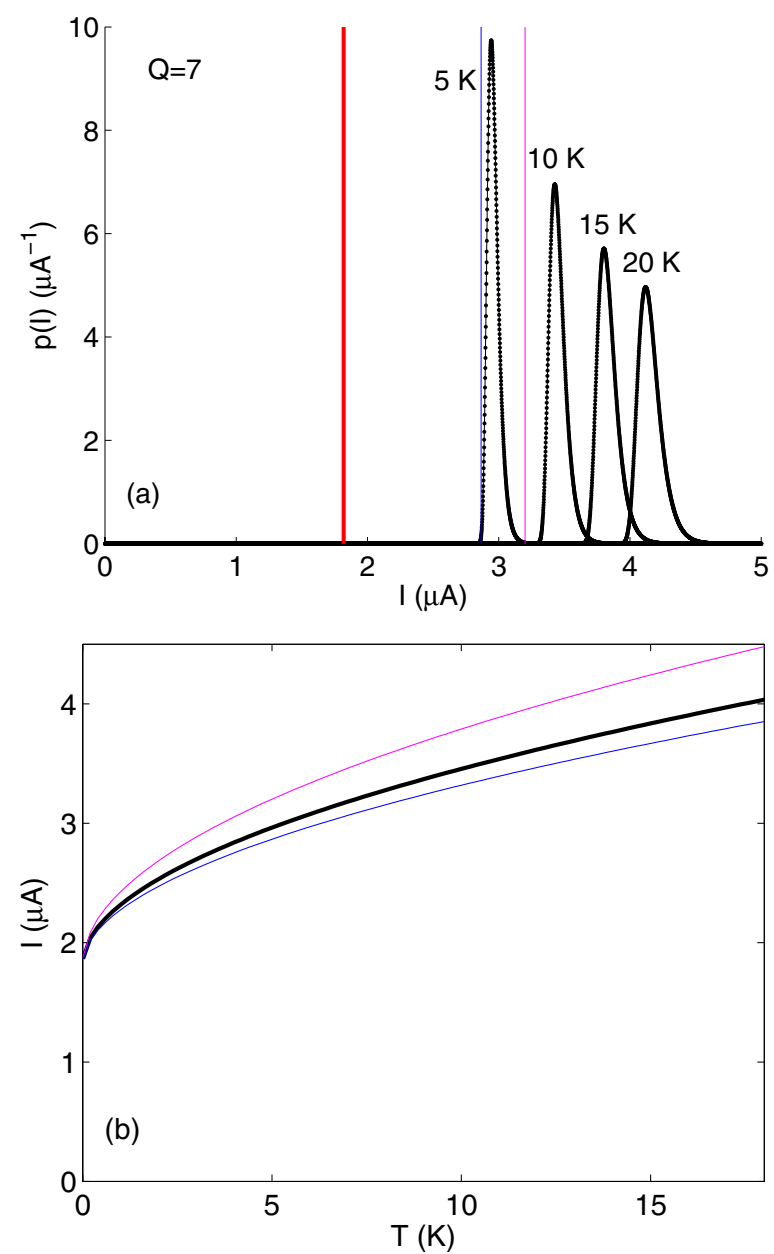

FIG. 9. (Color online) (a) Thermally activated return distributions at various temperatures, for $Q=7$. The distribution was constructed from the retrapping rate in Eq. (3) in the same way that Eq. (2) was used to construct the $p(I)$ distribution shown in Fig. 1. The vertical lines (pink and blue online) show the boundaries of the $5 \mathrm{~K}$ distribution, within which $99.99 \%$ of switching events occur. The thick vertical line (red online) shows the return current in the absence of fluctuations. (b) Variation with temperature of the mean return current (thick black line) and the top (pink online) and bottom (blue online) of the return-current distribution.

Figure 10 shows the variation in $I_{\mathrm{r}} / I_{\mathrm{sw}}$ with area at selected temperatures, assuming junctions remain in the underdamped regime. The junction parameters chosen might be appropriate for intrinsic Josephson junctions. For large-area junctions, $I_{\mathrm{r}} / I_{\mathrm{sw}} \approx I_{R} / I_{c}$. For junctions with area of $\sim 1 \mu \mathrm{m}^{2}, I_{\mathrm{r}} / I_{\mathrm{sw}}$ is significantly larger than $I_{R} / I_{c}$ and so in this case identifying $I_{\mathrm{r}} / I_{\mathrm{sw}}$ with $I_{R} / I_{c}$ to infer $Q$ will give a significant underestimate of $Q$. The inset of Fig. 10 shows the difference between the crudely inferred $Q$ and the true $Q$ at $4.2 \mathrm{~K}$ : the crudely inferred $Q$ is less than the actual $Q$. If measurements at higher temperatures were used, the discrepancy would be larger. Therefore it is important to account for the reduction by thermal fluctuations of the switching and return currents. We emphasize that, since we are treating $Q$ as a temperatureindependent quantity, this reduction in the hysteresis is purely a thermal effect. For junctions with area of $0.1 \mu \mathrm{m}^{2}$, it can be seen by comparing Figs. 1 and 9 that the under- 


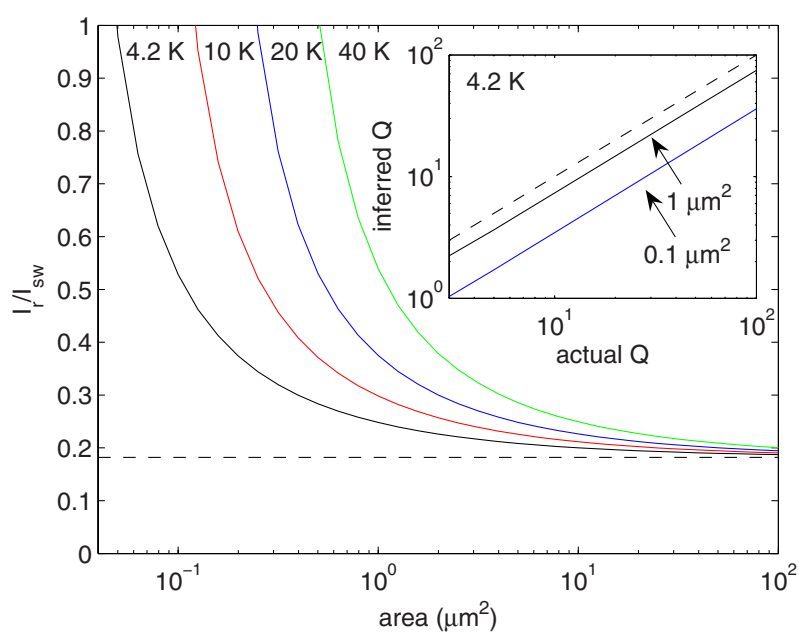

FIG. 10. (Color online) Area dependence of the ratio of mean return current and mean switching current for a quasi-dc measurement $d I / d t=10^{-7} \mathrm{~A} \mathrm{~s}^{-1}$. The dashed line shows the fluctuation-free value $I_{\mathrm{r}} / I_{\mathrm{sw}}=I_{R} / I_{c}=4 / \pi Q$. Inset: Variation in inferred $Q$ $=4 I_{\mathrm{sw}} / \pi I_{r}$ at $4.2 \mathrm{~K}$ with $Q$ for two junction areas. The dashed line shows $Q_{\text {inferred }}=Q_{\text {actual }}$.

damped return current and underdamped switching current become similar around $14 \mathrm{~K}$ and at higher temperatures the underdamped return current exceeds the underdamped switching current. This would imply that there is a current range $I_{\mathrm{sw}}<I<I_{\mathrm{r}}$, where neither the zero-voltage branch nor the resistive branch is stable: this is indeed a feature of the behavior we are describing in the temperature range $T$ $>T_{\text {low }}^{*}$ (for example, see Fig. 3). Above $T_{\text {low }}^{*}$, as we have discussed, it is necessary to consider, in addition, the effects of multiple escape and retrapping. Figure 11 shows the variation with temperature in the ratio $I_{\mathrm{r}} / I_{\mathrm{sw}}$ of these mean values. This ratio was obtained from simulations including the effects of both escape and retrapping as the current is ramped in either direction. At low temperatures we find $I_{\mathrm{r}} / I_{\mathrm{sw}}$ $\rightarrow 4 / \pi Q$. As the temperature is increased, even well below $T^{*}, I_{\mathrm{r}} / I_{\mathrm{sw}}$ departs significantly from $4 / \pi Q$. Around $T^{*}$, the distribution departs from its conventional thermally activated behavior. Hysteresis is still present for $T_{\text {low }}^{*}<T<T_{\text {high }}^{*}$ but to a decreasing extent as $T$ is increased. The inset of Fig. 11 shows the switching and return distributions for a temperature $14.5 \mathrm{~K}$ where $T_{\text {low }}^{*}<T<T_{\text {high }}^{*}$. The thick black curve shows that the mean and peak of the switching distribution lie above $I_{E R}$. The thick gray curve (red online) shows that the mean and peak of the return distribution lie below $I_{E R}$ (its width is related to the shape of the conventional return distribution). A difference in the mean switching and return currents, and so some hysteresis, persists even though the underdamped return current exceeds the underdamped switching current, which might be thought to imply the absence of hysteresis. The persistence in hysteresis above $T^{*}$ in the experiments in Refs. 3 and 5 is likely to be attributable to the distinction between $T^{*}$ and $T_{\mathrm{high}}^{*}$ : we expect hysteresis to persist up to $T \approx T_{\text {high }}^{*}>T^{*}$. For $T>T_{\text {high }}^{*}$, both escape and retrapping occur close to $I_{E R}$, so that $I_{\mathrm{r}} / I_{\mathrm{sw}} \rightarrow 1$ and hysteresis in the $I V$ characteristic is small.

We would like to note that the probability of a switch being counted is not the same as the probability of a single

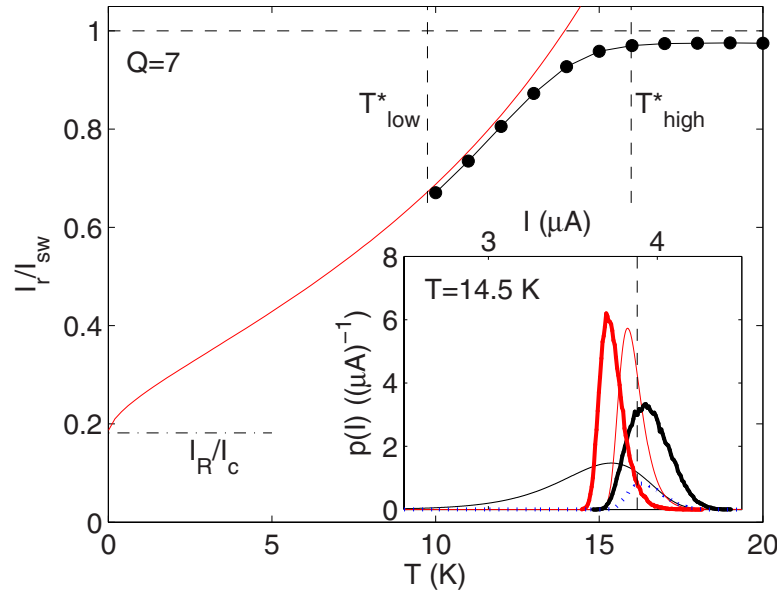

FIG. 11. (Color online) Temperature dependence of the ratio of mean return current and mean switching current for $d I / d t$ $=10^{-2} \mathrm{~A} \mathrm{~s}^{-1}$. The gray line (red online) shows the variation derived from the conventional thermal activation (Figs. 1 and 9) for $Q=7$. The black points show the dependence obtained from simulations including both escape and retrapping. The dashed horizontal line shows the value at which hysteresis disappears $I_{\mathrm{r}} / I_{\mathrm{sw}}=1$. Inset: Simulated switching distribution with current ramping up (heavy black curve) and simulated return distribution with current ramping down (heavy gray curve; red online) for $T=14.5 \mathrm{~K}$, demonstrating hysteresis in the switching and return currents. For comparison, the corresponding conventional thermally activated distributions are also shown [black and gray (red online) thin lines]. The broken vertical line shows the position of $I_{E R}$ and the dotted line (blue online) shows (the unrescaled) $p_{S} P_{n R}(I)$ from Ref. 5 for comparison. Compare with the fluctuation-free values, $I_{c}=10 \mu \mathrm{A}$ and $I_{R}$ $=4 I_{c} / \pi Q=1.82 \mu \mathrm{A}$.

escape not followed by a retrapping event, since a switch may be preceded by many escape-retrapping events. This difference was not appreciated in the quantitative analysis in Ref. 5, in which the latter quantity-although much smaller than the total probability of a switch-was evaluated as a function of the current of the initial escape (compare the dotted curve and the thick black line in the inset of Fig. 11) and then rescaled ${ }^{25}$ and fitted to experimental data.

\section{A. Frequency-dependent damping}

In our treatment so far, we have assumed that the damping $Q$ is frequency independent. Different retrapping and return behavior may arise when the damping is frequency dependent and we now turn to discuss these differences. If the damping of the system is frequency dependent-as is likely to be the case unless isolation resistors close to the junction are included or the shunt resistance is much less than the free-space impedance-the system will be characterized by much stronger damping shortly after escape than in the steady running state. This means that overdamped behavior at escape might lead to multiple escape-retrapping behavior shortly after an initial escape. However, once the junction has been in the running state for some time, it becomes much less strongly damped and might be characterized by the conventional underdamped dynamics and so is unlikely to be retrapped. 


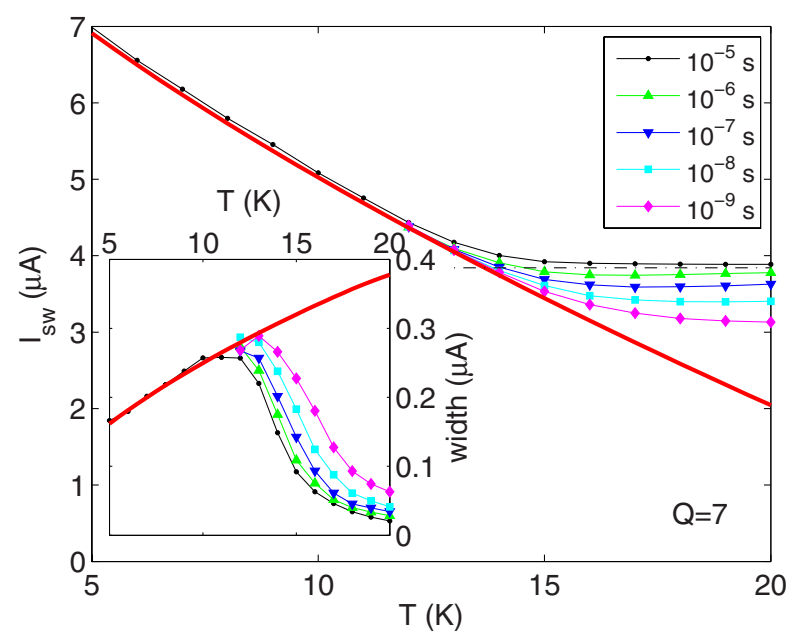

FIG. 12. (Color online) Variation in distribution characteristics with the time scale $1 / \nu_{c}$ for $Q=7$. Main panel: Mean switching current; the dash-dotted line shows $I_{E R}$. Inset: Switchingdistribution width. The lines joining points are guides to the eye. The lines without points (red online) show the conventional underdamped thermal behavior. Each data point corresponds to a simulated distribution based on 1000 switching events.

In their simulations, Kautz and Martinis ${ }^{2}$ considered a crossover to low-frequency damping once the junction has been mostly in the running state over a time scale $1 / \nu_{c}$, where $\nu_{c}$ is a characteristic frequency, a factor of $10^{2}-10^{5}$ smaller than the plasma frequency. In their simple model, which captures the qualitative features of the frequencydependent damping behavior, well above $\nu_{c}$ the damping is $Q_{1}$ and well below $\nu_{c}$ the damping is $Q_{0}<Q_{1}$.

In the simulations presented here so far, we counted a switching event if the junction was mostly in the running state over a certain time period. Experimentally, that time period might be determined by the response time of the measurement electronics. In the case of frequency-dependent damping, the time period is set instead by $1 / \nu_{c}$ since, once the junction has been mostly in the running state for the characteristic time $1 / \nu_{c}$ and becomes characterized by the much lighter damping $Q_{1}, \Gamma_{R}$ falls and retrapping becomes unlikely. This time is likely to be much shorter than the response time of the electronics.

To model, through simulations, the effect of a decrease in the damping at low frequencies, we neglect retrapping once the junction has been in the running state for a time $1 / \nu_{c}$. Figure 12 shows the variation in the mean and width of the switching distribution around $T^{*}$ as the characteristic time $1 / \nu_{c}$ varies. Note that the crossover temperatures $T_{\text {low }}^{*}$ and $T_{\text {high }}^{*}$ are essentially independent of the measurement time period, since they are set by the crossover between the extremes of the conventional underdamped distribution and by $I_{E R}$, all of which are essentially independent of the measurement time period. Figure 12 shows that a decrease in the characteristic time scale leads to a decrease in the mean switching current and to an increase in the width above $T^{*}$. The width and mean of the switching distribution for $T$ $>T_{\text {high }}^{*}$ therefore provide an indication of the crossover frequency $\nu_{c}$.
This variation with $\nu_{c}$ may be understood by considering the relative sizes of $\Gamma_{E}$ and $\Gamma_{R}$ in comparison to $\nu_{c}$ around the current at which switching occurs. In the multiple escape-retrapping regime, for small $\nu_{c}$ such that $\nu_{c}$ $\ll \Gamma_{E}, \Gamma_{R}$, the fraction of time in the running state $f_{r}$ is to a very good approximation $f_{r, \mathrm{eq}}=\Gamma_{E} /\left(\Gamma_{E}+\Gamma_{R}\right)$ and so switching is likely for $\Gamma_{E}=\Gamma_{R}$, i.e., for $I=I_{E R}$. (For the simulations presented in Sec. III, the same approximation holds for the temperatures of interest $T \approx T^{*}$, since the time period $\tau$ $=10^{-5} \mathrm{~s} \gg 1 / \Gamma_{E}, 1 / \Gamma_{R}$-compare with Fig. 6.) However for larger $\nu_{c}$ such that $1 / \nu_{c} \lesssim\left(1 / \Gamma_{E}+1 / \Gamma_{R}\right)$, large fluctuations of $f_{r}$ away from $f_{r, \text { eq }}$ occur. These fluctuations make switching likely at $I<I_{E R}$ and also increase the width of the switching distribution. The larger $\nu_{c}$ is, the smaller $I_{\mathrm{sw}}$ is likely to be. For example, in Fig. 3(a), for $1 / \nu_{c}=10^{-7} \mathrm{~s}$, the escape to the running state at around $3.603 \mu \mathrm{A}$ would last long enough to cause a switch, although $I_{E R}=3.8417 \mu \mathrm{A}$.

The presence of frequency-dependent damping also has a marked effect on the hysteresis of $I V$ measurements. For an initial escape as the current is ramped up from 0 , the system is characterized by the high-frequency damping $Q_{0}$. In contrast, when the current is ramped down from $I_{c}$, the system is characterized by the low-frequency damping $Q_{1}$. The more underdamped behavior for return means that hysteresis persists to much higher temperatures than for a system with frequency-independent damping $Q_{0}$.

\section{B. Application to previous work}

We now briefly discuss previous work in relation to frequency-dependent damping. The work of Krasnov et $a l .5,26$ was largely on samples with resistances $\sim 1 \Omega$, which are therefore likely to be characterized by approximately frequency-independent damping. However, the larger resistance of the IJJ samples suggests they may be affected by frequency-dependent damping and so the damping characterizing retrapping soon after escape is not the same as the damping extracted from the hysteresis in the $I V$ characteristics.

In their paper, Kivioja et al. ${ }^{4}$ considered mostly the switching dynamics, which are determined by the highfrequency damping, although the retrapping current $I_{m}$ is determined by the low-frequency damping. They did not include frequency-dependent damping in their modeling and they used the high-frequency $R$ as a fitting parameter. They expected the maximum in the width of the distribution to occur at $T_{d}$, when $\Gamma_{E}\left(I_{m}\right)$ corresponds to their experimental time scale, essentially equivalent to our $\Gamma_{I}$. This contrasts with our expectation for frequency-independent damping that at $T^{*}, I_{\mathrm{sw}}=I_{E R}$. Since $\Gamma_{E}\left(I_{m}\right)<\Gamma_{E}\left(I_{E R}\right)$, the crossover temperature $T^{*}<T_{d}$. Kivioja et al. extracted a value for $Q$ from their experimental results which was therefore larger than would be extracted if thermally activated retrapping had been included. This may explain the discrepancy between their extracted $Q=4.4$ and the $Q=4$ suggested by the nominal values of their experimental parameters.

Männik et $a l .{ }^{6}$ obtained values for the probability of retrapping from numerical Monte Carlo simulations and included frequency-dependent damping. This model has the 
advantage that it is able to account for the presumably initially increased rate of retrapping as, in the washboard ana$\log$, the particle first accelerates after escape. However, the results are less straightforward to analyze. The authors expressed the net escape rate as a sum of the probabilities of multiple escape-retrapping events, related to the thermal escape rate [our Eq. (2)] and the calculated retrapping probability. Although in detail the assessment of individual escape probabilities, a nontrivial problem, is oversimplified and relies on strictly inconsistent approximations, ${ }^{27}$ the model captures at least qualitatively well the effect of retrapping on the switching-current statistics. Männik et al. found good agreement between their model for the net escape rate and their experimental results. Their treatment of the probability of retrapping after escape as a time-independent quantity contrasts with the model of Ben-Jacob et al. ${ }^{7}$ in which retrapping is modeled by a rate [Eq. (3)] and so with a probability increasing linearly with time spent in the running state. The treatment of this probability as time independent by Männik et $a l .{ }^{6}$ is successful because in the model of frequency-dependent damping which they used, the time over which retrapping can occur is $\approx 1 / \nu_{c}$. Once the particle has been in the running state for a time $\gtrsim 1 / \nu_{c} \sim 1 / \omega_{P}$, lowfrequency (under)damping applies and retrapping is unlikely. This expectation is borne out by their simulations, in which they observed retrapping events only times $\lesssim 100 / \omega_{P}$ after escape. $^{28}$

\section{CONCLUSIONS}

In summary, we have presented discussion and simulations of the switching and return dynamics of moderately damped Josephson junctions. We emphasized that there is a regime in which the junction repeatedly escapes to and retraps from the running state. We also demonstrated through the use of simulations that, for some choices of parameters, the number of escapes and retraps during a single current ramp to an eventual switch into the running state may be very large $(\sim 10000)$. The multiple escape-retrapping regime, with a large number of escapes of duration $\sim 1 / \Gamma_{R}$, is intermediate between the underdamped regime, in which a single escape leads to switching, and the overdamped phasediffusion regime, in which a very large number of escapes of very short duration $\sim 1 / \omega_{P}$ may occur.

By examining the region around the crossover in the temperature dependence of the width in detail, we showed that the crossover is, in detail, described by not one but two crossover temperatures, $T_{\text {low }}^{*}$ and $T_{\text {high. }}^{*}$ The variations in the mean and width of the switching distribution (in the intermediate regime between the two crossovers) are largely determined by the shape of the thermally activated switching distribution. This shape therefore also determines the temperature of the maximum in the width, the quantity usually identified as the single crossover temperature. We showed that the shape of the switching distribution, parametrized by the skewness, indicates $T_{\text {low }}^{*}$ and $T_{\text {high }}^{*}$. We introduced a pertinent rate $\Gamma_{I}$ for understanding the dynamics. We showed that the details of the frequency dependence of the junction damping should affect measured values of the mean and width of switching distribution and weakly affect the crossover temperatures. We showed that the characteristic temperatures $T^{*}, T_{\text {low }}^{*}$, and $T_{\text {high }}^{*}$ are all dependent on the current-ramp rate and therefore are not uniquely determined by measurements at a particular ramp rate and in addition that there is some dependence of the behavior around and above $T^{*}$ on any frequency dependence of the damping.

We also considered the process of return to the supercurrent state as the current is ramped down in the presence of thermally activated retrapping events and the implications for measurements of hysteresis in moderately damped Josephson junctions. We found that some hysteresis is expected to persist above $T^{*}$, to $T_{\text {high }}^{*}$, even in junctions with frequency-independent damping. This suggests a resolution of the issue of hysteresis somewhat above $T^{*}$ in previous reports.

\section{ACKNOWLEDGMENTS}

The authors gratefully acknowledge financial support from the UK EPSRC. *j.fenton@ucl.ac.uk

${ }^{1}$ T. Fulton and L. Dunkleberger, Phys. Rev. B 9, 4760 (1974).

${ }^{2}$ R. Kautz and J. Martinis, Phys. Rev. B 42, 9903 (1990).

${ }^{3}$ A. Franz, Y. Koval, D. Vasyukov, P. Müller, H. Schneidewind, D. Ryndyk, J. Keller, and C. Helm, Phys. Rev. B 69, 014506 (2004).

${ }^{4}$ J. Kivioja, T. Nieminen, J. Claudon, O. Buisson, F. Hekking, and J. Pekola, Phys. Rev. Lett. 94, 247002 (2005).

${ }^{5}$ V. Krasnov, T. Bauch, S. Intiso, E. Hürfeld, T. Akazaki, H. Takayanagi, and P. Delsing, Phys. Rev. Lett. 95, 157002 (2005).

${ }^{6}$ J. Männik, S. Li, W. Qiu, Q. Chen, V. Patel, S. Han, and J. Lukens, Phys. Rev. B 71, 220509 (2005).

${ }^{7}$ E. Ben-Jacob, D. Bergman, B. Matkowsky, and Z. Schuss, Phys. Rev. A 26, 2805 (1982).

${ }^{8}$ Y. Chen, M. Fisher, and A. Leggett, J. Appl. Phys. 64, 3119
(1988).

${ }^{9}$ R. Cristiano and P. Silvestrini, J. Appl. Phys. 59, 1401 (1986).

${ }^{10} \mathrm{~J}$. Fenton and P. Warburton (unpublished).

${ }^{11}$ This period would be set by the details of an experiment-see Sec. IV A.

${ }^{12} \mathrm{M}$. Tinkham, Introduction to Superconductivity, 2nd ed. (McGraw-Hill, Singapore, 1996).

${ }^{13}$ The McCumber parameter $\beta_{c} \equiv Q^{2}$ is also sometimes used to characterize the damping.

${ }^{14}$ H. Kramers, Physica (Amsterdam) 7, 284 (1940).

${ }^{15}$ J. Fenton, M. Korsah, C. Grovenor, and P. Warburton, Physica C 460-462, 1470 (2007).

${ }^{16}$ Note that the numerator is the total probability of there having been no switch as the current ramps from 0 to $I$, so that the 
quotient represents the average probability per unit current of there having been no switch.

${ }^{17}$ See also the discussion in Sec. III.

${ }^{18}$ The counterintuitive nature of the accompanying decrease in the width with increasing temperature has previously been highlighted by Krasnov et al. in Refs. 5 and 26.

${ }^{19}$ V. Ambegaokar and A. Baratoff, Phys. Rev. Lett. 11, 104 (1963).

${ }^{20}$ Note that, even above $T_{\text {high }}^{*}$, zero voltage is expected for $I<I_{E I}$ and so the low-bias phase-diffusion voltage remains zero.

${ }^{21}$ Y. Ivanchenko and L. Zil'berman, Sov. Phys. JETP 28, 1272 (1969).

${ }^{22}$ H. Zappe, J. Appl. Phys. 44, 1371 (1973).

${ }^{23}$ Additionally, in a theoretical paper, Chen et al. (Ref. 8) showed that close to the fluctuation-free return current, the voltage departs from $V=I R$. However, we neglect that dependence here.
${ }^{24}$ M. Castellano, G. Torrioli, F. Chiarello, C. Cosmelli, and P. Carelli, J. Appl. Phys. 86, 6405 (1999).

${ }^{25}$ The distribution was scaled by dividing by the total probability of a switch following a single escape event.

${ }^{26}$ V. Krasnov, T. Golod, T. Bauch, and P. Delsing, Phys. Rev. B 76, 224517 (2007).

${ }^{27}$ For an escape event involving $n$ retraps before eventual escape in a time $\Delta t$, the average time for each escape is $\Delta t /(n+1)$, implying an equal probability of each escape event in that average time. However, the probabilities of the first $n$ escapes were set (Ref. 28) to 1, with the $(n+1)$ th escape being assigned the probability $\Gamma \Delta t /(n+1)$. More rigorously, the escape rate could have been expressed by integrating over all possible values of the time $t_{e}$ for each escape, subject to the constraint $\Sigma t_{e}=\Delta t$.

${ }^{28} \mathrm{~J}$. Männik (private communication). 